\title{
La epidemiología del dengue y del dengue hemorrágico en Santiago de Cuba, 1997
}

\author{
Luis Valdés, ${ }^{1}$ María G. Guzmán,, Gustavo Kourí, \\ Jorge Delgado, ${ }^{3}$ Isabel Carbonell, ${ }^{1}$ María V. Cabrera, ${ }^{1}$ \\ Delfina Rosario ${ }^{2}$ y Susana Vázquez ${ }^{2}$
}

RESUMEN En 1977, Cuba informó de su primera epidemia de dengue, durante la cual se registraron más de 500000 casos de fiebre del dengue causados por el serotipo 1 del virus. En 1981, se produjo una gran epidemia de dengue hemorrágico causada por el serotipo 2. Esa vez se notificaron 344203 casos en total, 10312 casos clínicos de dengue hemorrágico y 158 defunciones, de las cuales 101 correspondieron a niños. Por medio de una búsqueda activa con confirmación de laboratorio, en el municipio de Santiago de Cuba de la provincia del mismo nombre se detectó precozmente en enero de 1997 la reintroducción del dengue, especificamente del virus del tipo 2, genotipo Jamaica. En este trabajo se presentan los aspectos epidemiológicos de mayor interés referentes a esa epidemia. Se notificaron 3012 casos confirmados por serología, 205 clasificados como fiebre hemorrágica del dengue/sindrome de choque del dengue, de los cuales 12 fallecieron (todos adultos). La infección secundaria por virus del dengue fue uno de los principales factores de riesgo en el desarrollo de dengue hemorrágico y $98 \%$ de los casos, así como $92 \%$ de los fallecidos, manifestaron una respuesta de tipo secundario. Por primera vez se observó la posibilidad de contraer dengue hemorrágico en personas con una infección secundaria de 16 a 20 años después de la primera infección. Pertenecer a la raza blanca fue otro factor de riesgo de importancia, tal como se había observado desde la epidemia de 1981. En la última epidemia se demostró que la llamada "alerta de fiebre" no es útil para la detección temprana de una epidemia. Las medidas tomadas por las autoridades sanitarias del país evitaron la extensión de la epidemia a otros municipios que estaban infestados por Aedes aegypti.

En enero de 1997, Cuba notificó un brote de dengue 2 en el municipio de Santiago de Cuba (1). Este acontecimiento epidemiológico, de relevancia

\footnotetext{
1 Centro Provincial de Higiene y Epidemiología, Santiago de Cuba, Cuba.

2 Instituto de Medicina Tropical Pedro Kourí, Centro Colaborador OMS/OPS para Enfermedades Víricas. Toda correspondencia debe dirigirse a María G. Guzmán a la siguiente dirección: Centro Colaborador OMS/OPS para Enfermedades Víricas, Instituto de Medicina Tropical Pedro Kourí, Apartado 601, Marianao 13, La Habana, Cuba. Teléfono: 53-7-220450. Fax: 53-7-220633 y 53-7246051. Correo electrónico: lupe@ipk.sld.cu

3 Cuba, Ministerio de Salud Pública, Dirección Nacional de Epidemiología, La Habana, Cuba.
}

internacional, refleja una vez más la aparición y reaparición del dengue y de la fiebre hemorrágica del dengue (FHD) en la Región de las Américas e ilustra la necesidad de mantener una estrecha vigilancia para controlar los factores que influyen en su desarrollo. En el caso particular de Cuba, en la mayoría de los municipios se había logrado erradicar el vector o reducirlo a densidades que no permitían el desarrollo de un brote. En consecuencia, pasaron 15 años sin que se detectara la circulación de virus de dengue.

En 1977 ya se había informado en Cuba de una epidemia de fiebre del dengue (FD) causada por el serotipo 1, durante la cual se observaron más de medio millón de casos (2). Cuatro años después, en 1981, se produjo en la Región la primera epidemia de dengue hemorrágico, cuyo agente causal fue el serotipo 2 y en la cual se notificaron 344203 casos en total, 10312 casos de dengue hemorrágico y 158 defunciones (101 de ellas en niños) $(3,4)$. Esta epidemia se controló en poco más de 4 meses y a raíz de ella se estableció un programa de lucha para erradicar al vector Aedes aegypti (5), objetivo que se alcanzó en prácticamente todo el territorio nacional. 
En 1997, varios factores presentes en el municipio de Santiago de Cuba favorecieron la reinfestación y el incremento en densidad de A. aegypti, y la circulación del virus del dengue. Entre ellos, cabe destacar los siguientes: 1) El abasto de agua insuficiente a la ciudad de Santiago de Cuba obliga a almacenarla en las viviendas, habitualmente en recipientes deteriorados, que sirven de criaderos para los mosquitos. 2) El saneamiento ambiental es deficiente, lo que permite la acumulación de basuras y recipientes donde se crían los mosquitos. 3) La densidad de la población ha aumentado; Santiago de Cuba es el municipio más densamente poblado del país y $87 \%$ de los habitantes residen en la zona urbana, que cuenta además con un volumen importante de población en tránsito y con residencia temporal, factores que favorecen la transmisión de enfermedades. 4) En algunas zonas urbanas existen asentamientos residenciales en construcción, factores urbanísticos que facilitan la formación de criaderos potenciales de mosquitos (por ejemplo, en los sótanos de edificios donde viven varias familias) así como el surgimiento de barrios periurbanos con condiciones sanitarias y de urbanización inadecuadas. 5) Ha habido un incremento de migrantes, ya que la ciudad es un polo turístico importante para el área del Caribe y mantiene crecientes relaciones comerciales y culturales con otros países de la zona. Durante 1996 cerca de 800 naves, aviones y yates procedentes de países donde el dengue es endémico arribaron directamente a Santiago de Cuba. 6) Las tareas del programa de lucha y erradicación del vector se vieron deterioradas debido a una escasez de recursos financieros y fallas de la organización. 7) Hubo una disminución de la percepción del riesgo en la población, lo que influyó negativamente en la participación de la comunidad para eliminar los criaderos reales y potenciales del vector. 8) El bloqueo económico impuesto al país limitó la adquisición de recursos materiales (equipos e insumos) para la lucha contra $A$. aegypti y repercutió negativamente sobre la situación higiénicosanitaria y la salud de la población
$(6,7) .9)$ Se produjo una profunda crisis económica por el bloqueo (8) y el desplome del mundo socialista, con el que Cuba mantenía alrededor de $80 \%$ de su comercio exterior.

El objetivo del presente trabajo es brindar una caracterización detallada del brote de 1997 y llamar la atención a la comunidad científica internacional sobre el peligro que esta enfermedad representa para los países de las Américas, aun los que han logrado controlarla por largo tiempo.

\section{MATERIALES Y MÉTODOS}

\section{Área del estudio}

El municipio de Santiago de Cuba, perteneciente a la provincia del mismo nombre, se encuentra en la costa sur del extremo oriental del país, entre los $19^{\circ}$ $59^{\prime}$ y los $20^{\circ} 07^{\prime}$ de latitud norte y los $75^{\circ} 22^{\prime}$ y $76^{\circ} 57^{\prime}$ de longitud oeste. Predominan allí las temperaturas elevadas (30-32 ${ }^{\circ} \mathrm{C}$ durante todo el año) y un escaso régimen de lluvia. La población asciende a 475580 habitantes con una densidad de población de 453,2 por $\mathrm{km}^{2}$. La provincia de Santiago de Cuba logró eliminar a A. aegypti en 1987 y mantener esa condición hasta 1992, año en que se produjo la reinfestación. Paulatinamente, el vector fue extendiendo su distribución geográfica y aumentando en densidad a pesar de los esfuerzos realizados para controlarlo.

\section{Establecimiento de la vigilancia activa con confirmación de laboratorio}

Durante 1995 y, principalmente, en 1996 se trabajó de forma limitada en la lucha antivectorial y se había comenzado a ejecutar una estrategia de control atacando las zonas con los índices más altos de infestación por A. aegypti. No obstante, la situación vectorial era muy riesgosa, debido a que esos índices eran muy elevados en algunas zonas del municipio (índice/vivienda = 51 e índice de Breteau $=61,9$ ) y la actividad del dengue, muy intensa en la Región. Por otra parte, el sistema de vigilancia pasiva del dengue en este municipio era poco eficiente. Debido a la presencia de los factores de riesgo enumerados, los elementos que favorecían la reaparición del dengue y el peligro inminente de su reintroducción, se decidió implantar una vigilancia epidemiológica activa con confirmación de laboratorio. Siguiendo las pautas descritas en las guías publicadas por la OPS para la prevención y el control del dengue y el dengue hemorrágico en las Américas, el objetivo fue detectar los primeros casos que señalaran la reintroducción del dengue para poder establecer de forma inmediata las medidas adecuadas de control (9). El establecimiento de la vigilancia activa con confirmación de laboratorio en la provincia de Santiago de Cuba abarcó las siguientes acciones:

- Alertar al personal médico y a las unidades de salud del territorio sobre el peligro de la reaparición del dengue.

- Capacitar al personal del Laboratorio del Centro Provincial de Higiene, Epidemiología y Microbiología (CPHEM) de Santiago de Cuba en las técnicas serológicas de detección de anticuerpos IgM contra el dengue mediante pruebas ultramicroELISA (10).

- Buscar casos que correspondieran a la definición clínica descrita en las guías de la OPS, que identifican como caso sospechoso a todo paciente con un cuadro febril agudo, mialgias y artralgias, cefalea y dolor retroorbital acompañado o no de erupción cutánea y alguna manifestación hemorrágica (9).

- Establecer los criterios de caso probable (todo caso sospechoso en el que se demostrara la presencia de anticuerpos IgM contra el dengue mediante un sistema ultramicroELISA) y caso confirmado (todo caso probable confirmado por el laboratorio nacional de referencia del Instituto de Medicina Tropical Pedro Kourí (IPK) mediante la prueba ELISA de captación de IgM contra virus del dengue o en el que se realice el aislamiento viral o se detecte el ácido nucleico del virus) (9-11). 
Considerando que en Cuba por más de 15 años no había habido indicios de circulación de virus del dengue, todo caso con anticuerpos IgM detectados mediante la prueba ELISA de captación de IgM se consideraba un caso de dengue confirmado.

\section{RESULTADOS}

\section{Detección del brote}

La búsqueda de casos, que se intensificó a finales de enero de 1997, permitió que el día 29 de ese mes se confirmara el primer caso o caso índice de dengue. Los primeros síntomas se habían observado el día 22, es decir, 7 días antes. Se trataba de un paciente del sexo masculino, blanco, de 23 años de edad, trabajador no estatal, que no tenía antecedentes de viajes fuera de la localidad en la fecha probable de infección. Sus síntomas eran fiebre elevada acompañada de cefalea intensa, mialgias, dolor retroorbital, astenia y erupción. El paciente fue examinado por un médico del Hospital General Saturnino Lora, quien, sospechando que era un caso de dengue, informó al Departamento de Epidemiología del CPHEM de Santiago. Una muestra serológica del paciente dio resultados positivos a la prueba ultramicroELISA de detección de anticuerpos IgM contra el dengue, resultados que se confirmaron posteriormente mediante la prueba ELISA de captación de IgM.

El estudio epidemiológico del caso índice permitió confirmar clínica y serológicamente otros ocho casos de dengue relacionados con el mismo foco y reveló, además, que el caso primario se había manifestado el 4 de enero de 1997. La infección del paciente debe haber ocurrido durante la segunda quincena de diciembre de 1996.

El foco inicial surgió en el área de salud denominada "30 de Noviembre", la cual es una zona residencial ubicada en la parte noroeste de la ciudad de Santiago de Cuba con condiciones higiénicas y sanitarias satisfactorias caracterizadas por la buena calidad de las viviendas y sistemas adecuados de eliminación de líquidos residuales. No obstante, al igual que el resto de la ciudad, tiene un abastecimiento de agua deficiente que obligaba a almacenarla.
Los estudios epidemiológicos realizados en torno a los primeros casos, incluido el caso índice, no permitieron formar conclusiones con respecto al origen y las formas de introducción del virus. Sin embargo, en cuanto fue detectado el brote, se estableció una amplia política de internación inmediata de los pacientes con un cuadro clínico compatible con el dengue en hospitales ubicados en áreas exentas del vector, con objeto de romper la cadena de transmisión de la enfermedad.

En la figura 1 se presentan las secuencias temporal y espacial de los casos de dengue confirmados en Santiago de Cuba. En las semanas 4 a 6 del año, entre finales de enero y principios de febrero, se confirmaron 35 casos, todos localizados en el área de salud denominada "30 de Noviembre". Inicialmente, las acciones de lucha antivectorial se vieron limitadas por la falta de suficientes recursos como plaguicidas, motomochilas, personal con experiencia en las operaciones de fumigación y otros, lo que impidió abortar el brote a pesar de su temprana detección. Como puede observarse en la figura 1, para la semana 18 del año en

FIGURA 1. Secuencia de aparición de casos de dengue en las áreas de salud del municipio de Santiago de Cuba, $1997^{a}$

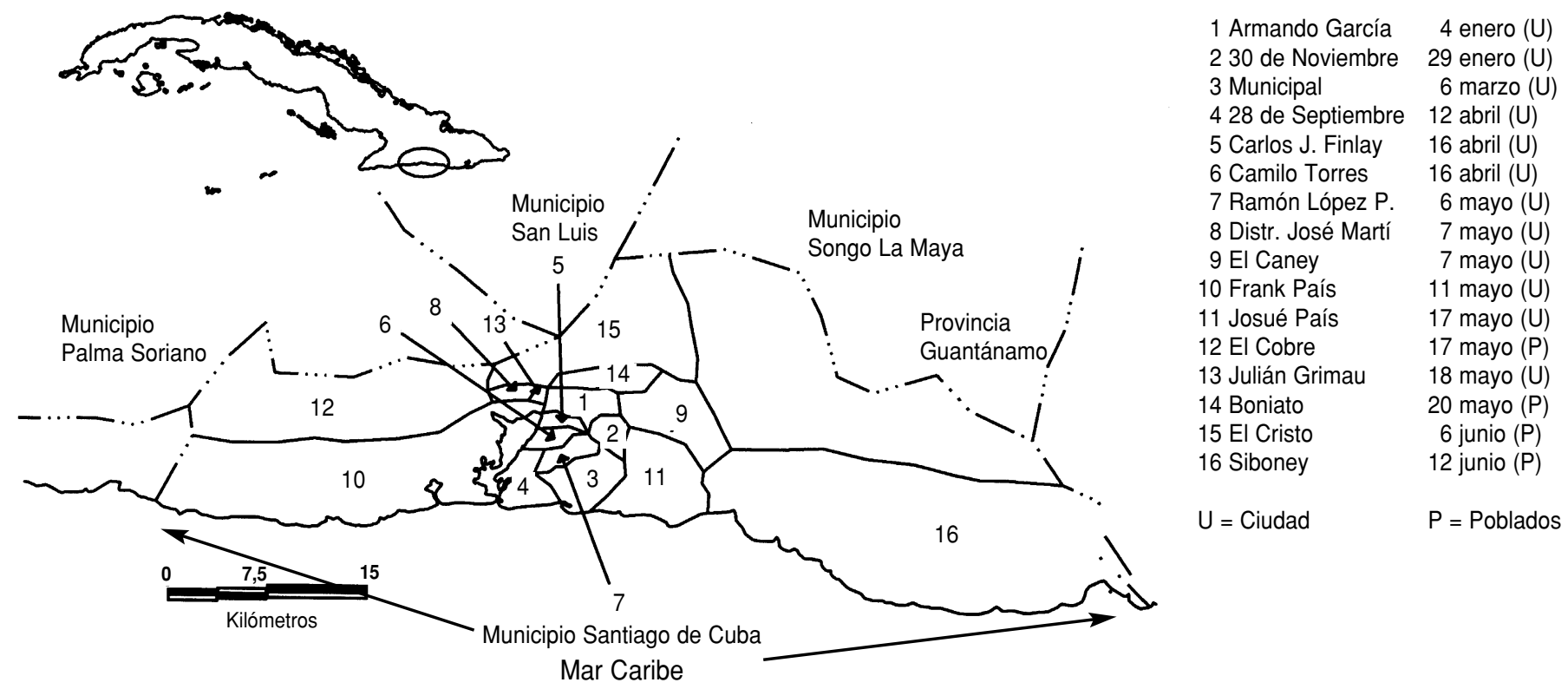

a Los números en el mapa representan la secuencia de aparición de los casos. 
el mes de mayo, casi todas las áreas de salud urbanas estaban notificando casos. Debe destacarse que a finales de ese mes ocurrió un fenómeno meteorológico caracterizado por intensas lluvias, que puede haber favorecido el incremento de la densidad del vector. La ciudad de Santiago de Cuba está rodeada de pequeños asentamientos semiurbanos llamados "poblados" en los cuales la notificación de casos de dengue por lo general tuvo lugar con posterioridad a la del área urbana de la ciudad. En el mes de mayo el incremento de la incidencia era ya evidente.

En el transcurso de la epidemia, el criterio de caso sospechoso se amplió a todo paciente con un cuadro febril agudo acompañado de alguna de las otras manifestaciones clínicas presentes en los casos de dengue según los criterios establecidos en las guías de la OPS (9). Esto se hizo con el fin de no pasar por alto ningún caso, aislar los casos en zonas exentas del vector, detectar la presencia de circulación viral en nuevas áreas y mantener una estrecha vigilancia clínica sobre los pacientes. Siguiendo este criterio, durante el brote se detectaron mediante búsqueda clínica activa un total de 17259 casos sospechosos. Se detectaron 757 casos febriles el 13 de junio, que constituyó el día de más alta incidencia. En 10024 de los casos sospechosos, la muestra serológica se obtuvo dentro de los primeros 5 a 10 días de haber comenzado la fiebre y se clasificaron como casos probables aquellos en los que se detectaron anticuerpos IgM contra el dengue mediante ultramicroELISA en el laboratorio de virología del CPHEM. Esas muestras se enviaron al laboratorio de referencia para confirmación y en 3012 pacientes $(30 \%)$ se verificó la presencia de anticuerpos IgM contra el dengue. Se clasificaron como casos de fiebre hemorrágica del dengue y síndrome de choque del dengue FHD/SCD (9) 205 pacientes, de los cuales fallecieron 12 (todos adultos). Para el mes de agosto la epidemia estaba controlada, aunque se produjeron con posterioridad algunos casos esporádicos. El último se notificó el 13 de noviembre. Aplicando el mismo porcentaje de confirmación serológica al número de pacientes sospechosos, podría inferirse que la epidemia produjo un total aproximado de 5186 enfermos de dengue.

Como consecuencia del bajo porcentaje de casos con confirmación serológica, se realizaron evaluaciones clínico-epidemiológicas rápidas en pacientes hospitalizados por diagnóstico presuntivo de dengue. Entre ellos se halló un porcentaje importante con manifestaciones respiratorias, por lo que se estudió una muestra al azar de 41 pares de sueros tomados de pacientes con cuadros clínicos compatibles con el dengue. El primer suero se tomó en la fase aguda y el segundo, en la fase convaleciente de la enfermedad. Los estudios serológicos para diagnosticar el dengue fueron negativos, pero se encontraron $12(29,3 \%)$ positivos a virus de la influenza A y B y adenovirus, con seroconversión o aumentos del título de anticuerpos al agente en cuestión de cuatro veces o más, según la técnica de inhibición de la hemaglutinación (información inédita, Laboratorio de Virus Respiratorios del IPK). Por último, durante esos meses, se detectó un brote de herpangina en más de 40 enfermos, que afectó fundamentalmente a niños de las áreas de salud "Municipal" y "López Peña". Se detectó asimismo un incremento de la

FIGURA 2. Incidencia de casos confirmados de dengue y dengue hemorrágico por semanas, municipio de Santiago de Cuba, 1997

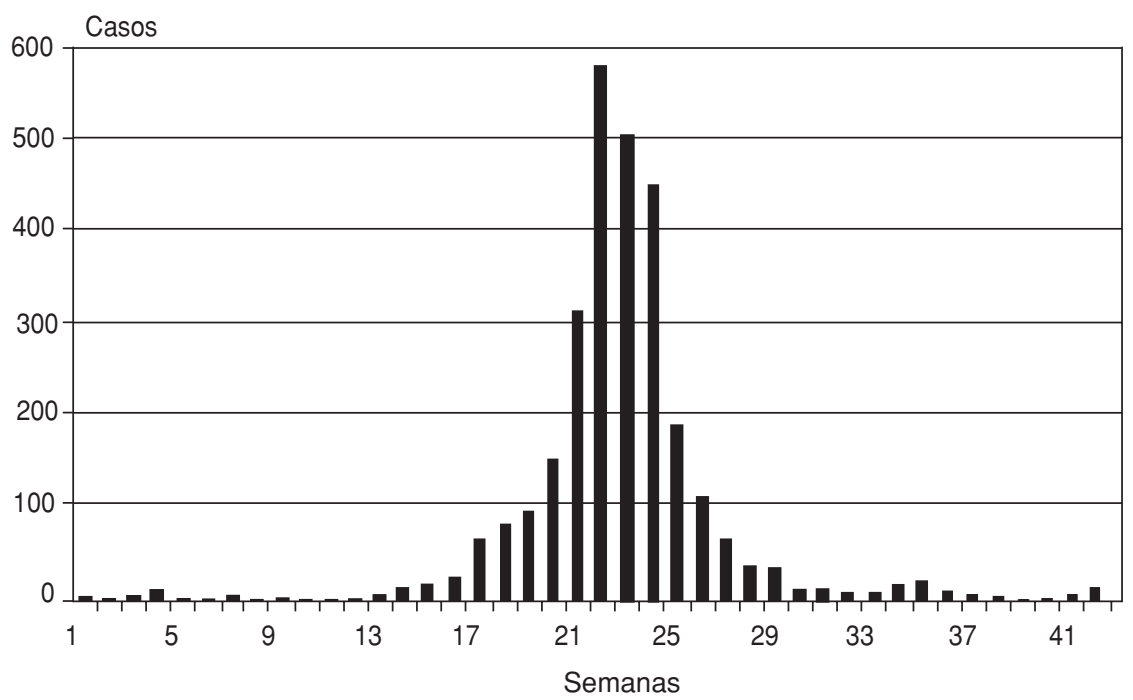

circulación de estreptococos, lo cual provocó un aumento de los casos de amigdalitis y escarlatina.

Según nuestra experiencia en Cuba, durante las epidemias de dengue pueden manifestarse otros procesos febriles que, teniendo en cuenta el carácter epidémico de esta enfermedad, aumentan falsamente la incidencia de casos de dengue. El presente estudio así lo sugiere y una cifra tan elevada como 29,3\% de los sospechosos mostró estar relacionada con infecciones respiratorias agudas. No obstante, la magnitud real de la infección por dengue se determinará por medio de un estudio seroepidemiológico retrospectivo que se encuentra actualmente en ejecución.

En la figura 2 se presenta la incidencia de casos de dengue confirmados semanalmente. Como puede observarse, el período de baja transmisión o, como se llama en inglés, lag phase (12), fue de aproximadamente 16 semanas antes de incrementarse el número de casos. La tasa de letalidad fue de 5,8 por 100 casos de FHD/SCD y de 0,4 por cada 100 casos de FD. La tasa de morbilidad de FHD/SCD fue de 43,1 por 100000 habitantes y representó $6,8 \%$ del total de casos de dengue confirmados serológicamente. De esta forma, por cada 14,7 casos confirma-

Semanas 
dos de FD se notificó un caso confirmado de FHD/SCD. De los casos de FD confirmados, $52,3 \%$ pertenecían al sexo femenino.

Es notable la mayor letalidad registrada en la epidemia de 1997 cuando se compara con la de 1981. Considerando la política amplia de hospitalización precoz, la elevada experiencia de los médicos en el manejo de los pacientes con la forma grave de la enfermedad y la disponibilidad de los recursos necesarios para la atención médica de los pacientes, no puede descartarse la posibilidad de que los casos fueran más graves esta vez. Algunos estudios en curso comparan la gravedad del cuadro clínico en los diferentes grupos de edad y cómo varió de una persona a otra según el momento en que cada una sufrió la infección secundaria en relación con la infección primaria. Finalmente, es importante señalar que todos los cálculos de los indicadores de la epidemia de 1981 se basaron en el estudio de pacientes diagnosticados clínicamente como casos de dengue o FHD y no en el estudio de casos confirmados serológicamente.

En el cuadro 1 puede observarse la distribución por grupos de edad de los casos confirmados en la epidemia de 1997. Las tasas más bajas correspondieron a los niños y los adultos mayores de 65 años. La baja incidencia en los niños podría estar relacionada con la mayor frecuencia de casos asintomáticos en ese grupo, mientras que entre los adultos de mayor edad es posible que hubiera un mayor número de personas con anticuerpos al virus del dengue 2 .

\section{Hallazgos clínicos principales}

El cuadro clínico predominante fue el de la FD, si bien 205 pacientes, todos adultos, fueron clasificados como casos de FHD/SCD (9). En una muestra de 352 pacientes adultos diagnosticados como casos de FD e internados en el Hospital General de Santiago, todos $(100 \%)$ tuvieron fiebre o antecedentes de fiebre. De ellos, $288(81,8 \%)$ tuvieron cefalea; $265(75,3 \%)$, mialgia; y 240 $(68,2 \%)$, artralgia. Solamente 30 personas $(8,5 \%)$ presentaron erupción cutánea. En 140 de los enfermos (39,7\%) se observó algún tipo de sangramiento menor como petequias, gingivorragias y epistaxis. El cuadro clínico de los pacientes de FD fue en general similar al descrito en la literatura, pero llamó la atención la presencia de hepatomegalia en $140(39,7 \%)$ de los casos $(9,13,14)$.

A continuación se presentan los síntomas y signos más frecuentemente observados en los 205 casos adultos de FHD/SCD. En todos ellos (100\%) se observó fiebre o antecedentes de fiebre y algún tipo de hemorragia. La trombocitopenia se verificó en $99 \%$ de los casos. En 187 (91,2\%) pacientes hubo cefalea, dolor abdominal en $176(86 \%)$ y mialgias en $154(75,1 \%)$. En $31(15,1 \%)$ de los casos se presentó choque y 12 de ellos fallecieron. La leucopenia afectó a $127(61,9 \%)$ pacientes; la ascitis a 141 $(68,7 \%)$ y el derrame pleural a 62 $(30,2 \%)$. En $131(67,1 \%)$ de 195 pacientes a los que se les realizó ultrasonido abdominal se detectó edema vesicular. Todos los casos $(100 \%)$ de FHD/ SCD presentaron hemoconcentración

CUADRO 1. Distribución por grupos de edad de los casos de dengue confirmados, municipio de Santiago de Cuba, 1997

\begin{tabular}{ccc}
\hline Grupo de edad (años) & No. de casos & Tasa por 100000 habitantes \\
\hline $0-4$ & 37 & 105,29 \\
$5-14$ & 158 & 219,01 \\
$15-24$ & 609 & 755,66 \\
$25-34$ & 969 & 1036,32 \\
$35-44$ & 582 & 938,10 \\
$45-64$ & 606 & 706,80 \\
$\geq 65$ & 51 & 142,25 \\
Total & 3012 & \\
\hline
\end{tabular}

demostrada por aumento del hematócrito (en $>20 \%$ ) o derrames en las membranas serosas. En 105 (51,2\%) se presentó hepatomegalia y en 78 $(38,0 \%)$, vómitos. El paciente más joven tenía 17 años y el más viejo, 66; la edad promedio fue de 34 años y la edad modal, de 27. En este grupo, 102 pacientes eran del sexo femenino y 103 del masculino.

\section{Estudios virológicos}

Durante el brote descrito, además de la vigilancia serológica se estableció la vigilancia virológica para detectar la posible introducción de otros serotipos. Tanto mediante métodos moleculares (15) como por aislamiento viral se comprobó que el virus dengue serotipo 2 era el agente etiológico de la epidemia. Este se detectó en muestras de suero de pacientes con FD y FHD/ SCD, así como en las vísceras de los fallecidos. A partir de 130 muestras de pacientes procedentes de 11 áreas de salud del municipio de Santiago de Cuba, se aislaron 46 cepas del virus. Mediante análisis de la secuencia nucleotídica de uno de los aislamientos, la cepa circulante se caracterizó como genotipo Jamaica, el cual ya se había visto asociado con FHD/SCD en la Región (16).

\section{Estudios retrospectivos}

Con objeto de conocer cuándo comenzó la epidemia, se revisaron los registros de los pacientes atendidos desde el 1 de noviembre de 1996 hasta el 28 de enero de 1997 en los servicios de urgencia de las unidades hospitalarias de la ciudad de Santiago de Cuba, donde se había atendido a más de 60000 personas. De los pacientes con signos y síntomas compatibles con el dengue (virosis, erupción, artralgia, mialgia, u otro), 592 podrían haber correspondido a casos sospechosos de dengue. Se visitó a esas personas en sus domicilios y, mediante una encuesta clínico-epidemiológica, el número se redujo a 154 debido a que los restantes no cumplían la definición de 
caso sospechoso de dengue. Se logró tomar muestras de sangre para estudios serológicos de 143 personas, en ninguna de las cuales se detectó la presencia de anticuerpos IgM específicos contra virus del dengue. Por otra parte, se revisaron también los registros de atención médica en las instalaciones turísticas ubicadas en áreas aledañas al foco inicial, pero no se encontraron casos sospechosos de dengue. Estos hallazgos, más los datos obtenidos por medio de la constante vigilancia seroepidemiológica de la rubéola y el sarampión -que permite examinar muestras serológicas de todo niño con fiebre y erupción-y los resultados de la vigilancia pasiva del dengue que se mantiene desde 1982 en todo el país, nos permitieron afirmar que la epidemia se había detectado en sus inicios.

\section{Organización de la atención médica durante el brote}

La decisión de hospitalizar a todos los casos sospechosos obedeció inicialmente al objetivo de aislar a los pacientes en áreas exentas del vector e interrumpir así la cadena de transmisión. Posteriormente, sirvió para mantener una estrecha vigilancia clínica sobre los pacientes así aislados, dada la posibilidad de que desarrollaran FHD/ SCD. Así, se internaron en unidades hospitalarias 13594 pacientes $(79,0 \%)$ $\mathrm{y}$ el resto se atendieron en la modalidad de internación domiciliaria, con el seguimiento y control de los médicos de familia. La atención médica se organizó en el nivel de la atención primaria con la asistencia de los médicos de familia, que realizaban la búsqueda activa de casos febriles y enfermos clasificados como sospechosos. Estas acciones permitieron el diagnóstico oportuno y la rápida internación de la mayoría de los casos. Se activaron y pusieron en marcha los cuatro policlínicos principales de urgencia de la ciudad y se reforzó el transporte sanitario para el traslado de pacientes. De un total de 2651 camas hospitalarias disponibles, se llegó a contar con 1655 para casos sospechosos de dengue. Además, se habilitaron dos instalacio- nes con 313 y 234 camas, respectivamente (el Politécnico de la Salud y el Hogar Materno Este) para la hospitalización de casos sospechosos. En total, llegó a disponerse de 202 camas para la internación de casos de dengue, $88 \%$ de ellas para adultos. En los dos hospitales generales de la ciudad, el Saturnino Lora y el General de Santiago de Cuba, se habilitaron salas de vigilancia y tratamiento para casos con signos de alarma de FHD/SCD (9), lo que permitió prevenir mediante una terapia hidratante la evolución hacia complicaciones y formas graves en cientos de pacientes. De igual forma se dispuso de cinco unidades de cuidados intensivos, una de ellas pediátrica, para la atención de enfermos graves.

\section{DISCUSIÓN}

\section{Factores de riesgo de la FHD/SCD}

Infección secundaria. Durante esta epidemia se comprobó que $98 \%$ de los casos de FHD/SCD presentaron infección secundaria, rasgo similar observado durante la epidemia de 1981 tanto en niños como en adultos (17-21). De 12 adultos fallecidos en 1997, 11 $(91,6 \%)$ tenían una infección secundaria. Durante la epidemia de 1981 señalamos que la infección secundaria se había producido entre 3 y 4 años después de la primaria. Asimismo, otros autores (22) han señalado que el intervalo de 6 meses a 5 años entre las dos infecciones constituye el mayor riesgo para que se produzca la FHD/SCD. Por estos motivos, no esperábamos una epidemia de dengue hemorrágico en Santiago de Cuba, quizá solamente algunos casos esporádicos. No obstante, esa no fue la situación y se produjeron 205 casos de FHD/SCD en una población en la que, sin lugar a dudas, hubo infección primaria por dengue 1 en 1977 y 1978 (aunque el serotipo 1 circuló hasta 1981, cuando fue desplazado por el 2), o sea, entre 16 y 20 años después de la primera infección. Esto sugiere que los anticuerpos "amplificadores" persisten y funcionan hasta 20 años después de la primera infección y quizá se mantengan de por vida.
Edad. Llamó la atención que todos los casos de FHD/SCD fueran adultos, ya que la forma grave de la enfermedad se ha descrito principalmente en niños en el sudeste asiático, aunque a partir de la epidemia cubana de 1981, se comenzaron a notificar cada vez con mayor frecuencia los casos graves en adultos, tanto en las Américas como en el sudeste asiático. El hecho de que no hubiera casos de FHD/SCD en niños se debe posiblemente a que las personas nacidas después de 1981 solo podían sufrir una infección primaria por dengue 2, ya que en el período de 1982 a 1996 no hubo circulación del dengue en Cuba y la infección secundaria es uno de los principales factores de riesgo de la forma grave de la enfermedad.

Raza. De 169 casos de FHD/SCD en los que se registró la raza según el color de la piel, 46,7\% eran blancos, $35,5 \%$ mestizos, $16,0 \%$ negros y solo $1,8 \%$, descendientes de la raza mongoloide. Esa distribución no guarda correspondencia con la estructura racial de la población de Santiago de Cuba, que se compone en $30,1 \%$ de blancos, $26,8 \%$ de negros, $42,8 \%$ de mestizos y $0,27 \%$ de raza mongoloide. Si se comparan los casos de FHD observados en Santiago de Cuba con los casos esperados según la estructura racial, se comprueba una diferencia estadísticamente significativa $\left(\chi^{2}=28,22 ; P<0,01\right)$. Según los datos analizados, la población blanca tiene un riesgo de padecer FHD 2,57 veces mayor que la población negra $(P<0,01)$ y 1,8 veces más que la mestiza $(P<0,01)$. Estas observaciones que sugieren un mayor riesgo de desarrollar FHD/SCD en la raza blanca cuando se compara con la negra coinciden con datos obtenidos durante la epidemia de 1981 (17, 18, 23). Entre los fallecidos se observó un predominio de los mestizos $(50,0 \%)$, seguidos de los blancos (33,3\%). Dos fallecidos eran de la raza negra, uno de ellos con rasgos de enfermedad drepanocítica $(16,6 \%)$. Es importante señalar que en la población de Santiago de Cuba no existe una mayor concentración de individuos de una raza específica por zonas o áreas geográficas determina- 
das, lo que sugiere que aparentemente tanto los blancos como los mestizos y negros tienen la misma posibilidad de tener contacto con el vector $y$, por ende, la misma posibilidad de adquirir la infección. No obstante, solo los estudios seroepidemiológicos en ejecución permitirán conocer si la tasa de infección por dengue fue similar en las personas de raza blanca, mestiza y negra. En estudios recientes realizados con voluntarios vacunados con virus dengue 2 atenuado, se ha observado que los blancos producen linfocitos $\mathrm{T}$ de memoria a un serotipo cruzado, a diferencia de los negros, que responden a un serotipo específico (24). Este hallazgo quizá implique que el fenómeno de inmunoamplificación viral es mayor en los blancos, lo que podría estar en concordancia con las observaciones epidemiológicas realizadas y explicar al menos en parte su mayor susceptibilidad de desarrollar FHD/SCD.

Enfermedades crónicas. Durante la epidemia de 1981, el asma bronquial, la drepanocitemia y la diabetes mellitus fueron factores de riesgo de FHD/ $\operatorname{SCD}(4,18)$. En el reciente brote pudo observarse entre los fallecidos un caso con antecedentes de asma bronquial y otro con rasgos drepanocíticos, lo que representa 8,3\%, cifra mucho mayor que la proporción de drepanocitemia observada en la población cubana. Llamó la atención que $97 \%$ de los casos con dengue hemorrágico eran hipertensos (personas con tensión arterial sistólica de 140 mm de Hg o más o tensión arterial diastólica de $90 \mathrm{~mm}$ de $\mathrm{Hg}$ o más o ambas cifras inclusive) y que $5,5 \%$ sufrían úlcera gastroduodenal, lo que sugiere la necesidad de realizar estudios de casos y controles en pacientes de dengue y dengue hemorrágico, apareando casos graves y no graves de dengue, y de establecer posibles asociaciones con esos estados patológicos.

Factores epidemiológicos. Los índices elevados de A. aegypti en Santiago de Cuba, la presencia de una elevada población susceptible tanto de sufrir una infección primaria como secunda- ria, y el intenso intercambio turístico y comercial con áreas endémicas crearon las condiciones necesarias para la aparición de un elevado número de casos de FD y FHD/SCD. Durante la epidemia se registraron índices de Breteau de más de 50 en seis de las 16 áreas de salud del municipio. Hacia el final de la misma, se verificó la transmisión en tres áreas de salud (Grimau, Caney y A. García) en las que los índices de viviendas estuvieron por debajo de 2 después del tratamiento focal con Abate y perifocal con fentión que se aplicó durante la epidemia y con posterioridad a ella. Por otra parte, para tener una idea del tamaño de la población susceptible de padecer dengue 2 en 1997, se extrapolaron a la población del municipio de Santiago de Cuba los valores de prevalencia de anticuerpos neutralizantes a dengue 1 y 2 obtenidos durante una encuesta seroepidemiológica realizada en 1983 en El Cerro, municipio densamente poblado con altas incidencias de la enfermedad durante las epidemias de 1977 y 1981 (23). De esta manera se pudo inferir que, en 1997, la población susceptible ascendía a 390094 personas de las cuales 120888 eran menores de 16 años, 181098 eran adultos que no habían padecido dengue con anterioridad y 88108 eran adultos que padecieron dengue en 1977 pero no en 1981 (susceptibles a una segunda infección). Esto significa que en Santiago de Cuba $82 \%$ de la población estaba en riesgo de contraer el dengue 2 y $18 \%$ de los adultos, en riesgo de desarrollar FHD/ SCD, que habitualmente se presenta durante la segunda infección.

Llama la atención que la mayoría de los casos de FHD/SCD (98\%) se concentraran en el 18\% de la población del municipio que se encontraba en riesgo de una segunda infección. Según Kourí et al. (17), para que se desarrolle una epidemia de FHD es necesario que concurran varios factores de riesgo del huésped, el agente y epidemiológicos. Entre estos últimos se destaca la necesidad de una amplia circulación viral, posiblemente responsable de que se infecte un mayor número de individuos con capacidad para desarrollar la segunda infección. De estos, la mayo- ría no evolucionarán hacia las formas graves de la enfermedad. Otros factores posiblemente dependientes del huésped (edad, sexo y raza entre otros) podrían determinar cuál individuo desarrollará el dengue hemorrágico. Finalmente resulta interesante comparar las epidemias de 1981 y de 1997 en cuanto a la relación de casos de FHD/SCD contra el total de individuos con posibilidad de desarrollar una infección secundaria. El porcentaje es parecido si se considera, como ya se ha hecho notar, que en ambas situaciones la mayoría de los casos graves constituían infecciones secundarias. Como resultado de la epidemia de dengue 1 en 1977, 44,46\% de la población cubana o 4446000 personas estaban en riesgo de sufrir una segunda infección (4). Durante la epidemia de 1981 se notificaron 10312 casos graves, que representaban $0,23 \%$ de la población. En la epidemia de 1997, el total de casos graves representó 0,23\% del grupo en riesgo de contraer una segunda infección por dengue.

Factores virales. Varios autores han señalado que la infección secundaria es el principal factor de riesgo de FHD/SCD y que dicho riesgo se incrementa 15 veces cuando el segundo serotipo es el dengue $2(17,25,26)$. Ambas condiciones se cumplieron en el caso de Santiago de Cuba. El serotipo 2 fue aislado tanto de pacientes de FD como de pacientes de FHD/SCD y fallecidos. El agente se clasificó como genotipo Jamaica, el cual se ha asociado a otros casos y epidemias de FHD en la Región (16).

Fue llamativo que el porcentaje de casos de FHD/SCD se incrementara a medida que la epidemia avanzaba a pesar de la experiencia adquirida por los médicos en el tratamiento de la enfermedad y la amplia política de hospitalización precoz. En mayo se notificaron 37 casos de FHD/SCD de un total de 705 casos confirmados en adultos, lo que dio una tasa de 5, 2\%. En junio la tasa fue de $7,39 \%(132 / 1785)$ y en julio, de $11,88 \%$ (29/244). Cuando se analizan los fallecidos por FHD/ $\mathrm{SCD}$ en relación con el total de casos 
de FHD/SCD y el total de casos de dengue confirmados por meses, también se observa un incremento estadísticamente significativo de las tasas a medida que la epidemia avanzaba (cuadro 2). Es importante señalar que el criterio de definición de casos de FHD/SCD no varió durante el transcurso de la epidemia.

Se ha planteado la posibilidad de que se produzca un incremento de la virulencia del agente a medida que este pasa por varios ciclos mosquitohombre-mosquito $(17,27,28)$. Nosotros observamos una situación similar en Cuba durante la epidemia de 1981 (17).

Resulta interesante que ni al inicio ni al final de la epidemia se notificaron casos de FHD/SCD. La ausencia de casos graves en esas etapas del brote podría explicarse, al menos en parte, por la falta de la intensa circulación viral que plantean varios autores como condición necesaria para el desarrollo de brotes de FHD (17). Finalmente, debemos destacar que en las áreas de salud J. Grimau, Boniato y Distrito José Martí, donde la aparición de casos fue más tardía, se registraron las tasas de incidencia de FHD/SCD más elevadas $(88,4 ; 81,6$ y 74,5 por 100000 habitantes, respectivamente).

\section{Control de la epidemia}

Al detectar el brote se hizo necesario trasladar las pocas fuerzas y recursos disponibles a nuevas zonas dentro del foco epidémico. Por el comportamiento inicial del mismo, se llegó a considerar que la epidemia se había abortado. Sin embargo, ya en la semana 16 del mes de abril la situación se tornó más compleja y planteó la necesidad de extender las medidas de lucha antivectorial a toda la ciudad de manera intensiva y con ciclos cortos de tratamiento adulticida. Para fines de mayo se habían asignado los fondos necesarios para la adquisición de equipos e insecticidas, se había capacitado al personal necesario y estaban en plena disposición equipos de alta productividad. A su vez, se realizaron jornadas de saneamiento ambiental e higienización, se protegieron los depósitos de agua y se logró una mayor participación de la comunidad en la fumigación y destrucción de criaderos intradomiciliarios. Por último, los médicos de familia brindaron audiencias sanitarias a la población mediante visitas y consultas.

La detección temprana de la epidemia desempeñó un papel importante en su limitación territorial al municipio de Santiago de Cuba y se logró mediante la vigilancia activa, ya que el indicador de "alerta de fiebre" no se manifestó hasta la semana 22 en el mes de mayo a pesar de que el virus estaba circulando desde diciembre de 1996. Este indicador no fue eficiente para la detección temprana y oportuna del brote ya que, si nos hubiéramos guiado por él, la extensión al resto de los municipios infestados por $A$. aegypti habría sido inevitable. En 1981 la epidemia se detectó en mayo, seis meses después de su inicio, cuando ya se había propagado por todo el país.

CUADRO 2. Incremento de la gravedad de los casos de dengue en los 3 meses de incidencia más alta de la enfermedad, municipio de Santiago de Cuba, 1997 ${ }^{\text {a }}$

\begin{tabular}{lrrrc}
\hline & Mayo & Junio & Julio & \\
\hline Casos de FHD/SCD $^{\text {b }}$ & 37 & 132 & 29 & - \\
Defunciones por FHD/SCD & 1 & 6 & 5 & - \\
Total de casos $^{\mathrm{c}}$ & 705 & 1785 & 244 & - \\
Casos de FHD/SCD (\%) $_{\text {Defunciones por FHD/SCD (\%) }}$ & 5,2 & 7,39 & 11,88 & $P<0,01$ \\
Defunciones/Total (\%) & 2,7 & 4,5 & 17,2 & $P<0,05$ \\
& 0,14 & 0,33 & 2,04 & $P<0,01$ \\
\hline
\end{tabular}

a Total de casos registrados en los meses de mayo, junio y julio.

b FHD = fiebre hemorrágica de dengue; SCD = síndrome de choque de dengue.

${ }^{c}$ Total de casos de dengue y dengue hemorrágico confirmados por serología.

Es importante señalar -aunque no forma parte de los objetivos del presente trabajo- que una vez detectado el brote en el municipio de Santiago de Cuba, se estableció la vigilancia activa con confirmación de laboratorio en todo el país y principalmente en 29 municipios de los 169 que tiene Cuba, que mostraban diversos grados de infestación. A su vez, se estableció otro laboratorio provincial para recibir y procesar las muestras de Ciudad Habana. El IPK actuó como laboratorio nacional de referencia y procesó un total de 9538 muestras para estudios serológicos procedentes de todo el país. Por medio de esta vigilancia activa se detectaron 17 casos de dengue en otras provincias, todos infectados en el municipio de Santiago de Cuba.

\section{CONCLUSIONES}

El brote de dengue de 1997 en el municipio de Santiago de Cuba demuestra una vez más que esta enfermedad reaparece en aquellas áreas donde las medidas de control del vector se deterioran. Cuba sigue manteniendo su política de erradicar el vector como forma de evitar nuevos brotes. A pesar de que este objetivo no se ha alcanzado, es evidente que desde el punto de vista epidemiológico el país se mantuvo libre de la enfermedad por más de 15 años.

Finalmente debemos señalar que si la epidemia cubana de 1981 marcó un hito en la historia del dengue en nuestra Región, la epidemia de 1997 fue un acontecimiento epidemiológico de primera prioridad que mostró cuán necesario es mantener las medidas de control vectorial y de vigilancia epidemiológica. Por otra parte, del estudio detallado y profundo de este fenómeno epidemiológico y específicamente de las relaciones entre la raza, la infección secundaria, el papel de los anticuerpos amplificadores de la infección (20 años después de la primera infección) y otros factores, surgirán nuevos conocimientos sobre la etiopatogenia de la enfermedad que aportarán datos de interés para su control. 


\section{REFERENCIAS}

1. Kourí G, Guzmán MG, Valdés L, Carbonell I, Rosario D, Vázquez S, et al. Reemergence of dengue in Cuba: a 1997 epidemic in Santiago de Cuba. Emerg Infect Dis 1998;4:89-92.

2. Pan American Health Organization. Dengue in the Caribbean, 1977. Washington, DC: PAHO; 1979. (Scientific Publication 375).

3. Kourí G, Más P, Guzmán MG, Soler M, Goyenechea A, Morier M. Dengue hemorrhagic fever in Cuba, 1981: rapid diagnosis of the etiologic agent. Bull Pan Am Health Organ 1983;17:126-132.

4. Kourí G, Guzmán MG, Bravo J, Triana C. Dengue haemorrhagic fever/dengue shock syndrome: lessons from the Cuban epidemic, 1981. Bull World Health Organ 1989;67: 375-380.

5. Armada JA, Figueredo R. Application of enviromental management principles in the programme for eradication of Aedes (Stegomyia) aegypti (Linnaeus-1762) in the Republic of Cuba-1984. Bull Pan Am Health Organ 1986; 20:186-193.

6. Anonymous. Editorial. Lancet 1996;348:1461.

7. Kirkpatrick A.F. Role of the USA in shortage of food and medicine in Cuba. Lancet 1996;348:1489-1491.

8. Garfield R, Santana S. The impact of the economic crisis and the U.S. embargo on health in Cuba. Am J Public Health 1997;87:1520.

9. Pan American Health Organization. Dengue and dengue hemorrhagic fever in the Americas: guidelines for prevention and control. Washington, DC: PAHO;1994. (Scientific Publication 548).

10. Laferté J, Pelegrino JL, Guzmán MG, González G, Vázquez S, Hermida C. Rapid diagnosis of dengue virus infection using a novel $10 \mu \mathrm{IgM}$ antibody capture ultramicroELISA assay (MAC UMELISA Dengue). Adv Modern Biotecnol 1992;1:19.4.

11. Vázquez S, Sáenz E, Huelva G, González A, Kourí G, Guzmán MG. Detección de IgM con- tra el virus del dengue en sangre entera absorbida en papel de filtro. Rev Panam Salud Publica 1998;3:174-178.

12. Gubler DJ. Surveillance for dengue and dengue hemorrhagic fever. Bull Pan Am Health Organ 1989;23:397-404.

13. Halstead SB. Dengue viruses. En: Gorbach SL, Bartlett JG, Blacklow NR, eds. Infectious Diseases. Philadelphia: Sanders 1992;1830-1835.

14. Halstead SB. Dengue hemorrhagic fever. En: Nelson WE, Vaughan VC, McKay RJ, eds. Textbook of pediatrics. 9a ed. Philadelphia: WB Saunders; 1969. Pp. 667-669.

15. Lanciotti, RS, Calisher CH, Gubler DG, Chang G, Vordam V. Rapid detection and typing of dengue viruses from clinical samples by using reverse transcriptase-polymerase chain reaction. J Clin Microbiol 1992;30:545-551.

16. Rico-Hesse R. Molecular evolution and distribution of dengue viruses type 1 and 2 in nature. Virology 1990;174:479-493.

17. Kourí G, Guzmán MG, Bravo J. Why dengue haemorrhagic fever in Cuba?: II, An integral analysis. Trans Royal Soc Trop Med Hyg 1987;81:821-823.

18. Bravo J, Guzmán MG, Kourí G. Why dengue haemorrhagic fever in Cuba?: I, Individual risk factors for dengue haemorrhagic fever/ dengue shock syndrome (DHF/DSS) in adults. Trans Royal Soc Trop Med Hyg 1987; 81:816-820.

19. Guzmán MG, Kourí G, Bravo J, Soler M, Martínez E. Sequential infection as risk factor for dengue hemorrhagic fever/dengue shock syndrome (DHF/DSS) during the 1981 dengue hemorrhagic Cuban epidemic. Mem Inst Oswaldo Cruz 1991;86:367.

20. Guzmán MG, Kourí G, Martínez E, Bravo J, Riverón R, Soler M, et al. Clinical and serological study of Cuban children with dengue hemorrhagic fever/dengue shock syndrome (DHF/DSS). Bull Pan Am Health Organ 1987; 21:270-278.
21. Díaz AG, Kourí G, Guzmán MG, Lobaina L, Bravo J, Ruiz A, et al. Description of the clinical picture of dengue hemorrhagic fever/ dengue shock syndrome (DHF/DSS) in adults. Bull Pan Am Health Organ 1988;22: 133-144.

22. Halstead SB. Observations related to pathogenesis of dengue hemorrhagic fever. Yale J Biol Med 1970;42:350-360.

23. Guzmán MG, Kourí G, Bravo J, Soler M, Vázquez S, Morier L. Dengue hemorrhagic fever in Cuba, 1981: a retrospective seroepidemiologic study. Am J Trop Med Hyg 1990; 42:179-184.

24. Dharakul T, Kurane I, Bhamarapravati N, Yoksan S, Vaughn DW. Dengue virus specific memory $\mathrm{T}$ cell responses in human volunteers receiving a live attenuated dengue virus type 2 candidate vaccine. J Infect Dis 1994;170: 27-33.

25. Halstead SB. Pathogenesis of dengue: challenges to molecular biology. Science 1988;239: 475-481.

26. Thein S, Min M, Shwe TN, Aye M, Zaw A, Aye $\mathrm{K}$, et al. Risk factors in dengue shock syndrome. Am J Trop Med Hyg 1997;56:566-572.

27. Anónimo. Dengue and dengue haemorrhagic fever: dengue serotype 2 epidemic, Townsville, Queensland. Wkly Epidemiol Rec 1993; 48:357-360.

28. Gubler DJ, Reed D, Rosen L, Hitchock JC. Epidemiologic, clinical and virologic observations on dengue in the Kingdom of Tonga. Am J Trop Med Hyg 1978;27:581-589.

Manuscrito recibido el 9 de abril de 1998 y aceptado para publicación, tras revisión, el 5 de febrero de 1999. 
A dengue epidemic that Cuba reported in 1997 registered more than 500000 cases of

The epidemiology of dengue and dengue hemorrhagic fever in Santiago de Cuba, dengue fever produced by viral serotype 1 . In 1981, there was an epidemic of dengue hemorrhagic fever produced by serotype 2 of the virus. This time 344203 clinical cases were reported, 10312 of which were severe cases of hemorragic fever that led to 158 fatalities (101 of them among children). The reintroduction of dengue, and specifically of dengue viral serotype 2 (Jamaica genotype), was quickly detected in January 1997 through an active surveillance system with laboratory confirmation of cases in the municipality of Santiago de Cuba, in the province of the same name. The main epidemiological features of this outbreak are reported in this paper. A total of 3012 cases were reported and serologically confirmed. These included 205 cases classified as dengue hemorrhagic fever/dengue shock syndrome (DHF/DSS), 12 of which were case fatalities (all among adults). Secondary infection with dengue virus was one of the most important risk factors for DHF/DSS. Ninety-eight percent of the DHF/DSS cases and $92 \%$ of the fatal cases had contracted a secondary infection. It was the first time dengue hemorrhagic fever was documented as a secondary infection 16 to 20 years after initial infection. Belonging to the white racial group was another important risk factor for DHF/DSS, as had been observed during the 1981 epidemic. During the most recent epidemic it was demonstrated that the so called "fever alert" is not useful for early detection of an epidemic. Measures taken by the country's public health officials prevented spread of the epidemic to other municipalities plagued by 